\title{
Three dimensional f-Kenmotsu manifold satisfying certain curvature conditions
}

\author{
Venkatesha And Divyashree G. \\ Department of Mathematics, \\ Kuvempu University, \\ Shankaraghatta - 577 451, Shimoga, Karnataka, INDIA. \\ vensmath@gmail.com, gdivyashree9@gmail.com
}

\begin{abstract}
The purpose of the present paper is to study pseudosymmetry conditions on f-Kenmotsu manifolds.
\end{abstract}

\section{RESUMEN}

El propósito del presente artículo es estudiar condiciones de pseudosimetría en variedades f-Kenmotsu.

Keywords and Phrases: f-Kenmotsu manifold, cyclic parallel Ricci tensor, almost pseudo Ricci symmetry, pseudosymmetry, Ricci pseudosymmetry, Ricci generalized pseudosymmetry.

2010 AMS Mathematics Subject Classification: 47A63; 47A99. 


\section{Introduction}

Let $M^{n}$ be an almost contact manifold with an almost contact metric structure $(\phi, \xi, \eta, g)[1]$. We denote by $\Phi$, the fundamental 2-form of $M^{n}$ i.e., $\Phi(X, Y)=g(X, \phi Y)$ for any vector fields $X, Y \in$ $\chi\left(M^{n}\right)$, where $\chi\left(M^{n}\right)$ being the Lie algebra of differentiable vector fields on $M^{n}$. Furthermore, we recollect the following definitions $[1,3,8]$.

The manifold $M^{n}$ and its structure $(\phi, \xi, \eta, g)$ is said to be:

i) normal if the almost complex structure defined on the product manifold $M^{n} \times R$ is integrable (equivalently, $[\phi, \phi]+2 \mathrm{~d} \eta \otimes \xi=0$ ),

ii) almost cosymplectic if $d \eta=0$ and $d \Phi=0$,

iii) cosymplectic if it is normal and almost cosymplectic (equivalently, $\nabla \phi=0$, where $\nabla$ is covariant differentiation with respect to the Levi-Civita connection).

The manifold $\mathrm{M}^{\mathrm{n}}$ is called locally conformal almost cosymplectic (respectively, locally conformal cosymplectic) if $M^{n}$ has an open covering $\left\{U_{t}\right\}$ endowed with differentiable functions $\sigma_{t}: U_{i} \longrightarrow R$ such that over each $U_{t}$ the almost contact metric structure $\left(\phi_{t}, \xi_{t}, \eta_{t}, g_{t}\right)$ defined by

$$
\phi_{\mathrm{t}}=\phi, \quad \xi_{\mathrm{t}}=e^{\sigma_{\mathrm{t}}} \xi, \quad \eta_{\mathrm{t}}=e^{-\sigma_{\mathrm{t}}} \eta, \quad g_{\mathrm{t}}=e^{-2 \sigma_{\mathrm{t}}} \mathrm{g}
$$

is almost cosymplectic (respectively, locally conformal cosymplectic).

Normal locally conformal almost cosymplectic manifold were studied by Olszak and Rosca [7]. An almost contact metric manifold is said to be f-Kenmotsu if it is normal and locally conformal almost cosymplectic. The same type of manifold was also studied by Yildiz et al. [9] using the projective curvature tensor. Olszak and Rosca [7] also gave a geometric interpretation of $f$ Kenmotsu manifolds and studied some curvature restrictions. Among others, they proved that a Ricci symmetric f-Kenmotsu manifold is an Einstein manifold.

Our work is structured in the following way: After introduction, we have given some basic equations of $\mathrm{f}$-Kenmotsu manifold in section 2. Section 3 deals with the study of 3-dimensional $\mathrm{f}$ Kenmotsu manifold with cyclic parallel Ricci tensor. And we study almost pseudo Ricci symmetric, pseudosymmetric, Ricci pseudosymmetric and Ricci generalized pseudosymmetric 3-dimensional fKenmotsu manifolds in sections 4, 5, 6 and 7, respectively.

\section{2 f-Kenmotsu manifolds}

Let $\mathrm{M}^{\mathrm{n}}$ be a smooth $(2 \mathrm{n}+1)$-dimensional manifold endowed with an almost contact metric structure $(\phi, \xi, \eta, g)$ which satisfy

$$
\phi^{2}=-i d+\eta \otimes \xi, \quad \eta(\xi)=1, \quad \eta \cdot \phi=0,
$$




$$
\phi \xi=0, \quad \eta(X)=g(X, \xi), \quad g(\phi X, \phi Y)=g(X, Y)-\eta(X) \eta(Y),
$$

for any vector fields $X, Y \in \chi\left(M^{n}\right)$ where $i d$ is the identity of the tangent bundle $T M^{n}, \phi$ is a tensor field of type $(1,1), \xi$ is a vector field, $\eta$ is a 1 -form and $g$ is a Riemannian metric.

We say that $\left(M^{n}, \phi, \xi, \eta, g\right)$ is an $f$-Kenmotsu manifold if the Levi-Civita connection $\nabla$ of $\phi$ satisfies the condition $[6]$

$$
\left(\nabla_{X} \phi\right)(Y)=f[g(\phi X, Y) \xi-\eta(Y) \phi X],
$$

where $f \in C^{\infty}\left(M^{n}\right)$ is strictly positive and $d f \wedge \eta=0$. If $f=0$, then the manifold is cosymplectic [5]. An $f$-Kenmotsu manifold is called regular if $f^{2}+f^{\prime} \neq 0$ where $f^{\prime}=\xi f$.

In an f-Kenmotsu manifold, from (2.3) we have

$$
\nabla_{X} \xi=f[X-\eta(X) \xi] .
$$

The condition $\operatorname{df} \wedge \eta=0$ holds if $\operatorname{dim} M^{n} \geq 5$ but it does not hold if $\operatorname{dim} M^{n}=3$ [7].

$$
\left(\nabla_{X} \eta\right)(Y)=f[g(X, Y)-\eta(X) \eta(Y)] .
$$

In a 3-dimensional Riemannian manifold, we have

$$
\begin{aligned}
R(X, Y) Z= & g(Y, Z) Q X-g(X, Z) Q Y+S(Y, Z) X-S(X, Z) Y \\
& -\frac{r}{2}\{g(Y, Z) X-g(X, Z) Y\} .
\end{aligned}
$$

In a 3-dimensional f-Kenmotsu manifold, we see that [7]

$$
\begin{aligned}
R(X, Y) Z= & \left(\frac{r}{2}+2 f^{2}+2 f^{\prime}\right)(X \wedge Y) Z-\left(\frac{r}{2}+3 f^{2}+3 f^{\prime}\right)\{\eta(X)(\xi \wedge Y) Z \\
& +\eta(Y)(X \wedge \xi) Z\}, \\
S(X, Y)= & \left(\frac{r}{2}+f^{2}+f^{\prime}\right) g(X, Y)-\left(\frac{r}{2}+3 f^{2}+3 f^{\prime}\right) \eta(X) \eta(Y),
\end{aligned}
$$

where R, S, Q and $r$ are the Riemannian curvature tensor, the Ricci tensor, the Ricci operator and the scalar curvature, respectively.

Now from(2.7), we have the following:

$$
\begin{gathered}
R(X, Y) \xi=-\left(f^{2}+f^{\prime}\right)[\eta(Y) X-\eta(X) Y], \\
R(\xi, Y) Z=-\left(f^{2}+f^{\prime}\right)[g(Y, Z) \xi-\eta(Z) Y], \\
\eta(R(X, Y) Z)=-\left(f^{2}+f^{\prime}\right)[g(Y, Z) \eta(X)-g(X, Z) \eta(Y)] .
\end{gathered}
$$

And from (2.8), we get

$$
S(X, \xi)=-2\left(f^{2}+f^{\prime}\right) \eta(X)
$$

and

$$
Q \xi=-2\left(f^{2}+f^{\prime}\right) \xi .
$$




\section{3-dimensional f-Kenmotsu manifold with cyclic parallel Ricci tensor}

Suppose the manifold $\mathrm{M}^{\mathrm{n}}$ under consideration satisfies the cyclic parallel Ricci tensor condition [4]. Then we have

$$
\left(\nabla_{X} S\right)(Y, Z)+\left(\nabla_{Y} S\right)(Z, X)+\left(\nabla_{Z} S\right)(X, Y)=0
$$

for all $X, Y, Z \in \chi\left(M^{n}\right)$.

From the above equation, it is seen that $r$ is constant. And we have

$$
\begin{aligned}
\left(\nabla_{X} S\right)(Y, Z)+\left(\nabla_{Y} S\right)(Z, X)+\left(\nabla_{Z} S\right)(X, Y)= & -\left(\frac{r}{2}+3 f^{2}+3 f^{\prime}\right)\left[\left(\nabla_{X} \eta\right)(Y) \eta(Z)\right. \\
& +\eta(Y)\left(\nabla_{X} \eta\right)(Z)+\left(\nabla_{Y} \eta\right)(Z) \eta(X) \\
& +\eta(Z)\left(\nabla_{Y} \eta\right)(X)+\left(\nabla_{Z} \eta\right)(X) \eta(Y) \\
& \left.+\eta(X)\left(\nabla_{Z} \eta\right)(Y)\right] .
\end{aligned}
$$

From (3.1) and (3.2), we get

$$
\begin{aligned}
& \left(\frac{r}{2}+3 f^{2}+3 f^{\prime}\right)\left[\left(\nabla_{X} \eta\right)(Y) \eta(Z)+\eta(Y)\left(\nabla_{X} \eta\right)(Z)+\left(\nabla_{Y} \eta\right)(Z) \eta(X)\right. \\
& \left.+\eta(Z)\left(\nabla_{Y} \eta\right)(X)+\left(\nabla_{Z} \eta\right)(X) \eta(Y)+\eta(X)\left(\nabla_{Z} \eta\right)(Y)\right]=0 .
\end{aligned}
$$

Using (2.5) in (3.3), we get

$$
\begin{aligned}
& \left(\frac{r}{2}+3 f^{2}+3 f^{\prime}\right)[g(X, Y) \eta(Z)+g(X, Z) \eta(Y)+g(Y, Z) \eta(X)+g(Y, X) \eta(Z) \\
& +g(Z, X) \eta(Y)+g(Z, Y) \eta(X)-6 \eta(X) \eta(Y) \eta(Z)]=0, \quad \text { since } f \neq 0 .
\end{aligned}
$$

On substituting $X=Y=e_{i}$ in (3.4), where $e_{i}$ is an orthonormal basis of the tangent space at each point of the manifold and taking summation over $i, 1 \leq i \leq 3$, which gives

$$
4\left\{\frac{r}{2}+3 f^{2}+3 f^{\prime}\right\} \eta(Z)=0 .
$$

Hence, we get $\eta(Z)=0$, which is a contradiction. Therefore, from (3.5) we have

$$
r=-6\left(f^{2}+f^{\prime}\right)
$$

Conversely, if $r=-6\left(f^{2}+f^{\prime}\right)$ then from (3.2), we obtain

$$
\left(\nabla_{X} S\right)(Y, Z)+\left(\nabla_{Y} S\right)(Z, X)+\left(\nabla_{Z} S\right)(X, Y)=0 .
$$

From the above discussions we have the following:

Theorem 3.1. A 3-dimensional f-Kenmotsu manifold satisfies cyclic parallel Ricci tensor if and only if the scalar curvature $\mathrm{r}=-6\left(\mathrm{f}^{2}+\mathrm{f}^{\prime}\right)$, provided $\mathrm{f} \neq 0$. 


\section{Almost pseudo Ricci symmetric 3-dimensional f-Kenmotsu manifold satisfying cyclic Ricci tensor}

Chaki and Kawaguchi [2] introduced the concept of almost pseudo Ricci symmetric manifolds as an extended class of pseudo symmetric manifolds. A Riemannian manifold $\left(M^{n}, g\right)$ is called an almost pseudo Ricci symmetric manifold $(A P R S)_{\mathfrak{n}}$, if its Ricci tensor $S$ of type $(0,2)$ is not identically zero and satisfies the following condition

$$
\left(\nabla_{\mathrm{U}} \mathrm{S}\right)(\mathrm{V}, \mathrm{W})=[\mathrm{A}(\mathrm{U})+\mathrm{B}(\mathrm{U})] \mathrm{S}(\mathrm{V}, \mathrm{W})+\mathrm{A}(\mathrm{V}) \mathrm{S}(\mathrm{U}, \mathrm{W})+\mathrm{A}(\mathrm{W}) \mathrm{S}(\mathrm{U}, \mathrm{V}),
$$

where $A$ and $B$ are two non-zero 1-forms defined by

$$
A(U)=g\left(U, P_{1}\right), B(U)=g\left(U, P_{2}\right) .
$$

By taking the cyclic sum of (4.1), we see that

$$
\begin{aligned}
& \left(\nabla_{\mathrm{u}} \mathrm{S}\right)(\mathrm{V}, \mathrm{W})+\left(\nabla_{\mathrm{V}} \mathrm{S}\right)(\mathrm{W}, \mathrm{U})+\left(\nabla_{\mathrm{W}} \mathrm{S}\right)(\mathrm{U}, \mathrm{V})=[3 \mathrm{~A}(\mathrm{U})+\mathrm{B}(\mathrm{U})] \mathrm{S}(\mathrm{V}, \mathrm{W}) \\
& +[3 \mathrm{~A}(\mathrm{~V})+\mathrm{B}(\mathrm{V})] \mathrm{S}(\mathrm{U}, \mathrm{W})+[3 \mathrm{~A}(\mathrm{~W})+\mathrm{B}(\mathrm{W})] \mathrm{S}(\mathrm{U}, \mathrm{V}) .
\end{aligned}
$$

Let $M^{n}$ admit a cyclic Ricci tensor, then (4.3) becomes

$$
\begin{aligned}
& {[3 A(U)+B(U)] S(V, W)+[3 A(V)+B(V)] S(U, W)+} \\
& {[3 A(W)+B(W)] S(U, V)=0 .}
\end{aligned}
$$

Replacing $W$ by $\xi$ in the above equation and using (2.12) and (4.2), we get

$$
\begin{aligned}
& -\left\{2\left(f^{2}+f^{\prime}\right)\right\}[3 A(U)+B(U)] \eta(V)-\left\{2\left(f^{2}+f^{\prime}\right)\right\}[3 A(V)+B(V)] \eta(U) \\
& +\left[3 \eta\left(P_{1}\right)+\eta\left(P_{2}\right)\right] S(U, V)=0 .
\end{aligned}
$$

In (4.5), substituting $\mathrm{V}=\xi$ and using (2.12) and (4.2), we have

$$
-\left\{2\left(f^{2}+f^{\prime}\right)\right\}[3 A(U)+B(U)]-4\left\{2\left(f^{2}+f^{\prime}\right)\right\}\left[3 \eta\left(P_{1}\right)+\eta\left(P_{2}\right)\right] \eta(U)=0 .
$$

Again treating $\mathrm{U}$ by $\xi$ and using (4.2) in (4.6), we obtain

$$
\left\{f^{2}+f^{\prime}\right\}\left[3 \eta\left(P_{1}\right)+\eta\left(P_{2}\right)\right]=0,
$$

which implies

$$
\left[3 \eta\left(P_{1}\right)+\eta\left(P_{2}\right)\right]=0,
$$

since $\left\{\mathbf{f}^{2}+\mathbf{f}^{\prime}\right\} \neq 0$.

From (4.8) and (4.6), it follows that

$$
3 \mathrm{~A}(\mathrm{U})+\mathrm{B}(\mathrm{U})=0
$$

Thus, we can state:

Theorem 4.1. There is no almost pseudo Ricci symmetric 3-dimensional f-Kenmotsu manifold admitting cyclic Ricci tensor, unless 3A + B vanishes everywhere. 


\section{$5 \quad$ Pseudosymmetric 3-dimensional f-Kenmotsu manifold}

Let $M^{n}$ be an pseudosymmetric 3-dimensional f-Kenmotsu manifold. Then we have,

$$
(R(X, Y) \cdot R)(U, V) W=f_{R} Q(g, R)(U, V, W ; X, Y),
$$

for all $X, Y, U, V, W \in \chi\left(M^{n}\right)$.

From the above relation it follows that

$$
\begin{aligned}
& R(X, Y) R(U, V) W-R(R(X, Y) U, V) W-R(U, R(X, Y) V) W \\
& -R(U, V) R(X, Y) W=f_{R}\left[\left(X \wedge_{g} Y\right) R(U, V) W-R\left(\left(X \wedge_{g} Y\right) U, V\right) W\right. \\
& \left.-R\left(U,\left(X \wedge_{g} Y\right) V\right) W-R(U, V)\left(X \wedge_{g} Y\right) W\right],
\end{aligned}
$$

where

$$
\left(X \wedge_{g} Y\right) Z=g(Y, Z) X-g(X, Z) Y
$$

Substituting $X$ by $\xi$ and using (2.10) and (5.3), (5.2) yields

$$
\begin{aligned}
& {\left[\left(f^{2}+f^{\prime}\right)+f_{R}\right]\{g(Y, R(U, V) W) \xi-\eta(R(U, V) W) Y-g(Y, U) R(\xi, V) W} \\
& +\eta(U) R(Y, V) W-g(Y, V) R(U, \xi) W+\eta(V) R(U, Y) W-g(Y, W) R(U, V) \xi \\
& +\eta(W) R(U, V) Y\}=0 .
\end{aligned}
$$

Taking inner product of (5.4) with $\xi$, we get

$$
\begin{aligned}
& {\left[\left(f^{2}+f^{\prime}\right)+f_{R}\right]\{R(U, V, W, Y)-\eta(Y) \eta(R(U, V) W)-g(Y, U) \eta(R(\xi, V) W)} \\
& +\eta(U) \eta(R(Y, V) W)-g(Y, V) \eta(R(U, \xi) W)+\eta(V) \eta(R(U, Y) W) \\
& -g(Y, W) \eta(R(U, V) \xi)+\eta(W) \eta(R(U, V) Y)\}=0 .
\end{aligned}
$$

By using (2.11), (5.5) becomes

$$
\begin{aligned}
& {\left[\left(f^{2}+f^{\prime}\right)+f_{R}\right]\left\{R(U, V, W, Y)-\left(f^{2}+f^{\prime}\right)[-g(V, W) \eta(Y) \eta(U)\right.} \\
& +g(U, W) \eta(Y) \eta(V)-g(Y, U) g(V, W)+g(Y, U) \eta(V) \eta(W)+g(V, W) \eta(U) \eta(Y) \\
& -g(Y, W) \eta(U) \eta(V)-g(Y, V) \eta(W) \eta(U)+g(Y, V) g(U, W)+g(Y, W) \eta(V) \eta(U) \\
& -g(U, W) \eta(Y) \eta(V)+g(V, Y) \eta(W) \eta(U)-g(U, Y) \eta(V) \eta(W)]\}=0 .
\end{aligned}
$$

Contracting the above equation, we obtain

$$
\left[\left(f^{2}+f^{\prime}\right)+f_{R}\right]\left\{S(V, W)+2\left(f^{2}+f^{\prime}\right) g(V, W)\right\}=0 .
$$

The above equation can hold only if either

(i) $\left(f^{2}+f^{\prime}\right)=-f_{R}$, or 
(ii) $S(V, W)=\alpha g(V, W)$, where $\alpha=-2\left(f^{2}+f^{\prime}\right)$.

This leads to the following:

Theorem 5.1. A 3-dimensional pseudosymmetric f-Kenmotsu manifold with never vanishing function $\left\{\left(\mathrm{f}^{2}+\mathrm{f}^{\prime}\right)=-\mathrm{f}_{\mathrm{R}}\right\}$ is an Einstein manifold.

\section{Ricci pseudosymmetric 3-dimensional f-Kenmotsu mani- fold}

Suppose $\left(M^{n}, g\right)$ be a 3-dimensional Ricci pseudosymmetric f-Kenmotsu manifold. Then we have,

$$
(R(X, Y) \cdot S)(U, V)=f_{S} Q(g, S)(U, V ; X, Y)
$$

for all $X, Y, U, V, W \in X\left(M^{n}\right)$. From the above relation it follows that

$$
(R(X, Y) \cdot S)(U, V)=f_{S}\left(\left(X \wedge_{g} Y\right) \cdot S\right)(U, V),
$$

or

$$
\begin{aligned}
& -S(R(X, Y) U, V)-S(U, R(X, Y) V)=f[-g(Y, U) S(X, V)+g(X, U) S(Y, V) \\
& -g(Y, V) S(U, X)+g(X, V) S(U, Y)] .
\end{aligned}
$$

Replacing $\mathrm{X}$ and $\mathrm{U}$ by $\xi$ and using $(2.1),(2.10)$ and (2.12) in the above equation, we get

$$
\left[\left(f^{2}+f^{\prime}\right)+f_{S}\right]\left\{S(Y, V)+2\left(f^{2}+f^{\prime}\right) g(Y, V)\right\}=0,
$$

which follows that either $\left[\left(f^{2}+f^{\prime}\right)+f_{S}\right]=0$ or

$$
S(Y, V)=\alpha g(Y, V)
$$

where $\alpha=-2\left(f^{2}+f^{\prime}\right)$.

Thus we can state:

Theorem 6.1. If a 3-dimensional $\mathrm{f}$-Kenmotsu manifold $\mathrm{M}^{\mathrm{n}}$ is Ricci pseudosymmetric with restrictions $\mathrm{X}=\mathrm{U}=\xi$, then either $\left[\left(\mathrm{f}^{2}+\mathrm{f}^{\prime}\right)+\mathrm{f}_{\mathrm{S}}\right]=0$ or the manifold is an Einstein manifold.

\section{Ricci generalized pseudosymmetric 3-dimensional f-Kenmotsu manifold}

Consider a Ricci generalized pseudosymmetric 3-dimensional f-Kenmotsu manifold. Then we have

$$
(R(X, Y) \cdot R)(U, V) W=f\left(\left(X \wedge_{S} Y\right) \cdot R\right)(U, V) W
$$


for all $X, Y, U, V, W \in X\left(M^{n}\right)$.

We can write the above form as

$$
\begin{aligned}
& R(X, Y) R(U, V) W-R(R(X, Y) U, V) W-R(U, R(X, Y) V) W \\
& -R(U, V) R(X, Y) W=f[S(Y, R(U, V) W) X-S(X, R(U, V) W) Y \\
& -S(Y, U) R(X, V) W+S(X, U) R(Y, V) W-S(Y, V) R(U, X) W \\
& +S(X, V) R(U, Y) W-S(Y, W) R(U, V) X+S(X, W) R(U, V) Y]
\end{aligned}
$$

On substituting $\mathrm{X}=\mathrm{U}=\xi$, and using (2.10) and (2.12), (7.2) reduces to

$$
\begin{aligned}
& -\left(f^{2}+f^{\prime}\right)\left[\left(f^{2}+f^{\prime}\right) g(V, W) Y+R(Y, V) W-\left(f^{2}+f^{\prime}\right) g(Y, W) V\right] \\
& =f\left[\left(f^{2}+f^{\prime}\right) S(Y, V) \eta(W) \xi-2\left(f^{2}+f^{\prime}\right)^{2} g(V, W) Y-2\left(f^{2}+f^{\prime}\right) R(Y, V) W\right. \\
& +2\left(f^{2}+f^{\prime}\right)^{2} g(Y, W) \eta(V) \xi+\left(f^{2}+f^{\prime}\right) S(Y, W) \eta(V) \xi-\left(f^{2}+f^{\prime}\right) S(Y, W) V \\
& \left.+2\left(f^{2}+f^{\prime}\right)^{2} g(V, Y) \eta(W) \xi\right] .
\end{aligned}
$$

Taking inner product of the above equation with $\mathbf{Z}$, we get

$$
\begin{aligned}
& -\left(f^{2}+f^{\prime}\right)\left[\left(f^{2}+f^{\prime}\right) g(V, W) g(Y, Z)+g(R(Y, V) W, Z)-\left(f^{2}+f^{\prime}\right) g(Y, W) g(V, Z)\right] \\
& =f\left[\left(f^{2}+f^{\prime}\right) S(Y, V) \eta(W) \eta(Z)-2\left(f^{2}+f^{\prime}\right)^{2} g(V, W) g(Y, Z)\right. \\
& -2\left(f^{2}+f^{\prime}\right) g(R(Y, V) W, Z)+2\left(f^{2}+f^{\prime}\right)^{2} g(Y, W) \eta(V) \eta(Z) \\
& +\left(f^{2}+f^{\prime}\right) S(Y, W) \eta(V) \eta(Z)-\left(f^{2}+f^{\prime}\right) S(Y, W) g(V, Z) \\
& \left.+2\left(f^{2}+f^{\prime}\right)^{2} g(V, Y) \eta(W) \eta(Z)\right] .
\end{aligned}
$$

Contracting (7.4) and simplifying gives

$$
\left(f^{2}+f^{\prime}\right)(3 f-1)\left[S(Y, Z)+2\left(f^{2}+f^{\prime}\right) g(Y, Z)\right]=0,
$$

which means that either $\left(f^{2}+f^{\prime}\right)(3 f-1)=0$ or $S(Y, Z)=\alpha g(Y, Z)$, where $\alpha=-2\left(f^{2}+f^{\prime}\right)$. Hence we can state the following:

Theorem 7.1. If a 3-dimensional f-Kenmotsu manifold is Ricci generalized pseudosymmetric then either

(i) $\left(f^{2}+f^{\prime}\right)(3 f-1)=0$, or

(ii) it is an Einstein manifold.

Acknowledgement: The second author is thankful to UGC for financial support in the form of Rajiv Gandhi National Fellowship (F1-17.1/2015-16/RGNF-2015-17-SC-KAR-26367).

\section{References}

[1] D.E Blair, Contact manifolds in Riemannian geometry, Lecture Notes in Mathematics, 509, Springer-Verlag, Berlin-New York, 1976. 
[2] M.C. Chaki and T. Kawaguchi, On almost pseudo Ricci symmetric manifolds, Tensor N. S. 68, 10-14, 2007.

[3] Goldberg S. I. and Yano K, Integrability of almost cosymplectic structures, Pacific J. Math., 31, 373-382, 1969.

[4] A. Gray, Two classes of Riemannian manifolds, Geom. Dedicata. 7, 259-280, 1978.

[5] Janssens D and Vanhecke L, Almost contact structures and curvature tensors, Kodai Math. J. 4(1), 1-27, 1981.

[6] Olszak Z, Locally conformal almost cosymplectic manifolds, Colloq. math. 57, 73-87, 1989.

[7] Olszak Z and Rosca R, Normal locally conformal almost cosymplectic manifolds, Publ. Math. Debrecen. 39, 315-323, 1991.

[8] Sasaki S. and Hatakeyama Y, On differentiable manifolds with certain structures which are closely related to almost contact structures II, Tohoku Math. J., 13, 281-294, 1961.

[9] Yildiz A, De U.C. and Turan M, On 3-dimensional f- Kenmotsu manifolds and Ricci solitons, Ukrainian Math. J., 65(5), 684-693, 2013. 